\title{
Electromechanical Numerical Analysis of an Air-Core Pulsed Alternator via Equivalent Network Formulation
}

\author{
A. Musolino ${ }^{1}$, M. Raugi ${ }^{1}$, R. Rizzo ${ }^{1}$, L. Sani ${ }^{1}$ and V. Di Dio ${ }^{2}$ \\ ${ }^{1}$ DESTEC, Department of Energy, Systems, Territory and Construction Engineering, University of Pisa, Pisa, Italy; \\ ${ }^{2}$ DEIM, Department of Energy, Engineering Information and Mathematical Model, University of Palermo, Palermo, Italy
}

\begin{abstract}
In this paper the numerical analysis on an air-core pulsed alternator is presented. Since compulsators are characterized by very fast electromechanical transients, their accurate analysis requires strong coupling between the equations governing the electrical and the mechanical behavior. The device is investigated by using a dedicated numerical code capable to take into account eddy currents, compensating windings as well as the excitation/control circuits. Furthermore, the code is capable of modeling centrifugal forces and vibrations acting on the shaft due to electric and mechanical unbalances or to misalignments of the shaft from its centered position. This makes the code a very powerful tool during the design phase. Examples of application of the code to machines with non-uniform compensating shield and with shorted compensating windings are shown.
\end{abstract}

Index Terms - Air core machine, compulsator, numerical models, integral formulation, coupled analysis, eddy currents.

\section{INTRODUCTION}

The feeding of rail launchers requires the availability of generators able to supply extremely intense currents, often of impulsive waveform. The air-core pulsed alternator seems to be one of the most promising technology in the field of electromagnetic launchers power supplies [1-4]. These systems are made of non-ferromagnetic materials (usually with very high specific strength) and, in comparison with the iron-core generators, they need very high current to produce the proper exciting field [5]. On the contrary, due to the low values of inductances, the peak value of the output pulsed current can be increased. Compared to the iron-core generators, the maximum speed of an air-core rotor can reach higher values, increasing the energy storage ratio. Although self-excitation mode is usually adopted in these systems, some different excitation strategies were proposed to increase their performances [6], [7].

The traditional approach to the analysis of the air-core pulsed alternator is based on the standard mathematical model of rotating machines [8-10]. Unfortunately, these models disregard many real effects (eddy currents on the conductive media, interaction between primary and compensating windings, and so forth) allowing only simplified analyses. Other approaches [11], even though capable to perform a more accurate model, are not able to simulate complex phenomena like eddy currents or the velocity skin effect in the brushes system. Moreover, the use of combined numerical/analytical model with offline calculation of the parameters cannot simulate the real effects that take place in some parts of the system (non-uniform shield, shorted windings, and so forth).

The aim of this paper is to present a numerical analysis of an air-core pulsed alternator. This is achieved by adapting a research code, previously developed by the authors for research purpose.

The numerical code, named "EN4EM" (Equivalent Network for Electromagnetic Modeling), is able to simulate such kind of devices taking into account the following real characteristics:

- complex armature windings;

- the presence of excitation/control circuits;

- the eddy currents in all the conducting parts of the machine (the uniform or non-uniform shield, the shaft, and so forth);

- $\quad$ the compensating windings of different shapes and arrangements (aluminum sheet, single shorted turns, and so forth.);

- real winding turns connections;

- end-turn effects;

- relative movement between conductors;

Moreover, the numerical formulation can model centrifugal forces and vibrations acting on the shaft due to electric and mechanical unbalances or to misalignments of the shaft from its centered position, as well as the electromechanical transient behavior of the machine.

Finally, the possibility to analyze the entire system composed of the air-core compulsator and of the railgun, simulating the 3D coupled electromechanical transient behavior, makes the code a very powerful tool during the design phase of such devices.

\section{NUMERICAL ANALYSIS}

The code is based on an integral 3-D formulation that reduces the electromagnetic field analysis to an equivalent network analysis. During the modeling phase, only the active regions, the conductors and the magnetic materials (if present), are discretized by using elementary volumes, and an equivalent network is built. The currents in the branches of this equivalent network are straightforwardly mapped onto the currents flowing 

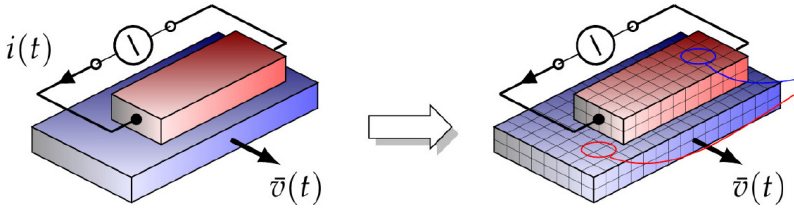

Fig. 1 Arrangement of stationary (blue) and moving (red) conductors.

in the elementary volumes. The relative motion induced electromotive force is taken into account by the $\boldsymbol{v} \times \boldsymbol{B}$ term while the force on current carrying conductor is obtained by the $\boldsymbol{j} \times \boldsymbol{B}$ term. The formulation is here briefly summarized; the details are reported in [12] and [13].

Let us consider two bodies in relative motion and magnetically interacting (see fig. 1); they are discretized with $n_{1}$ and $n_{2}$ elementary volumes. A $3-\mathrm{D}$ grid is obtained by connecting the centers of nearby elements. Subsequently a new elementary volume (evidenced in light red in fig. 2) is associated to each segment of the grid. These new elementary volumes carry uniformly distributed currents in the direction of the associated segment. Let $N_{1}$ and $N_{2}$ the numbers of conductive elements so obtained in the two bodies. Ohm's law is written at a point $P$ inside the k-th elementary conductive volume:

$\rho \boldsymbol{J}_{k}(P, t)=-\nabla V_{k}(P, t)-\frac{\partial}{\partial t} \boldsymbol{A}_{k}(P, t)+\boldsymbol{v}_{k}(P, t) \times \boldsymbol{B}_{k}(P, t)$

where $\boldsymbol{J}_{k}(P, t)$ is the current density, $-\nabla V_{k}(P, t)$ is the irrotational component of the electric field, $\boldsymbol{A}_{k}(P, t)$ is the vector potential, $\rho$ is the resistivity and $\boldsymbol{v}_{k}(P, t) \times \boldsymbol{B}_{k}(P, t)$ represents the motional term of the electric field.

By the additive property, we can write:

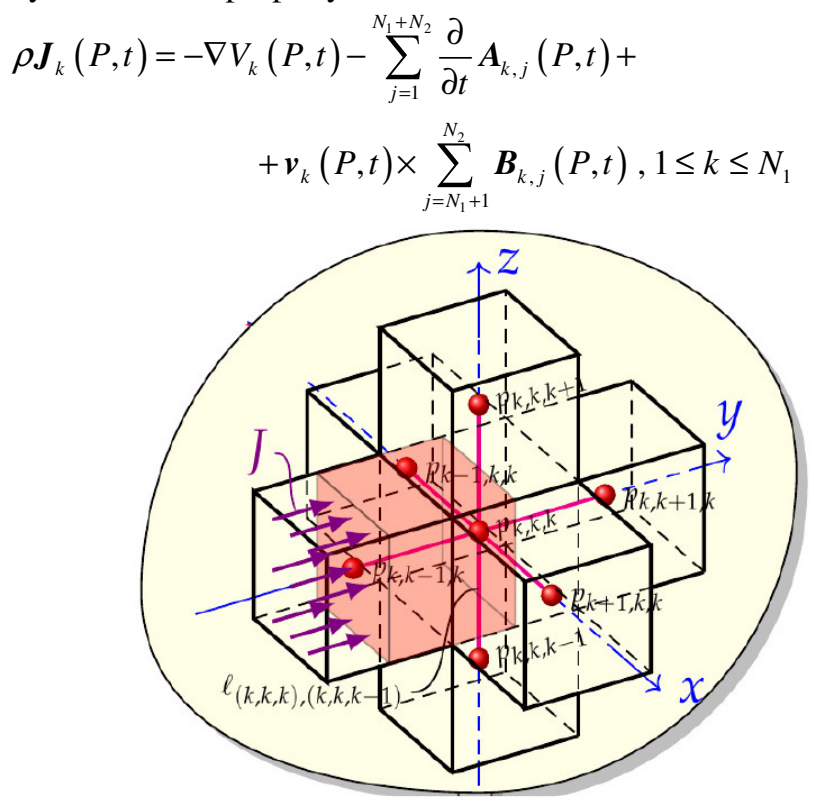

Fig. 2 The $3 \mathrm{D}$ grid and the elementary volumes associated to the grid.

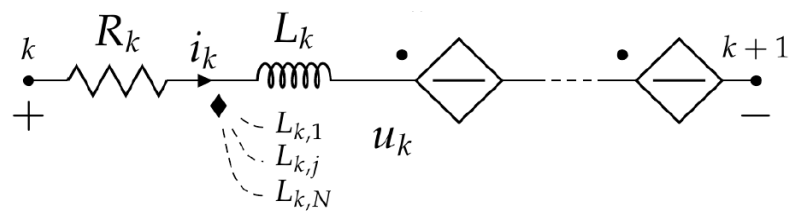

Fig. 3 A branch of the equivalent electric network.

where $\boldsymbol{A}_{k, j}(P, t)$ and $\boldsymbol{B}_{k, j}(P, t)$ are respectively the vector potential and the magnetic flux density produced in $P$ inside the $\mathrm{k}$-th volume by the current in the $\mathrm{j}$-th volume. Similarly when $N_{1}+1 \leq k \leq N_{1}+N_{2}$.

The above equation is projected on the corresponding segment of the grid and is averaged on the cross section. It yields:

$R_{k} i_{k}(t)+\sum_{j=1}^{N_{1}+N_{2}} L_{k, j} \frac{d}{d t} i_{j}(t)+\sum_{j=N_{1}+1}^{N_{2}} K_{k, j} i_{j}(t)=u_{k}(t), 1 \leq k \leq N_{1}$

This is the governing equation of a branch, which is a series connection of a resistor, an inductor coupled with the inductors in the other branches and a controlled voltage generator which takes into account the motional effects (fig. 3).

Fig. 4 reports an example of the equivalent network, built by the described formulation. It is solved by the mesh analysis:

$\boldsymbol{R} \boldsymbol{i}+\boldsymbol{L} \frac{d}{d t} \boldsymbol{i}+\boldsymbol{K i}=\boldsymbol{u}$

where $\boldsymbol{i}$ is the vector of the currents on the chords and $\boldsymbol{u}$ is the vector of the imposed e.m.f. acting in the meshes. Because of the relative movement, some of the elements of the matrices $\boldsymbol{L}$ and $\boldsymbol{K}$ change and have to be evaluated at every time step.

Once the currents are evaluated, the force on the $\mathrm{k}$-th volume due to current on the $\mathrm{j}$-th elements is written as:

$\boldsymbol{f}_{j k}=\int_{\Gamma_{k}} \boldsymbol{j}_{k} \times \boldsymbol{B}_{k, j} d \Gamma$

where $\boldsymbol{j}_{k}$ is the current density and the and $\boldsymbol{B}_{k, j}$ is the flux density in the $\mathrm{k}$-th volume produced by the current flowing in the $\mathrm{j}$-th one.

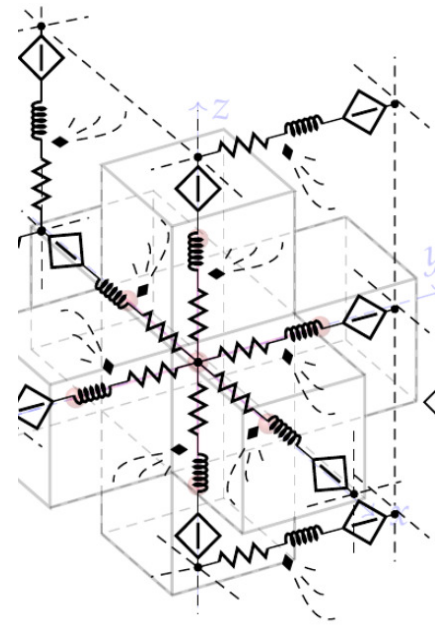

Fig. 4. A sample of the equivalent network. 
The governing equation of the coupled electromechanical system with 6 Degree of Freedoms (DoFs) is written as:

$$
\begin{aligned}
& \boldsymbol{L}(\boldsymbol{\xi}) \frac{d \boldsymbol{i}}{d t}+[\boldsymbol{R}(\boldsymbol{\xi})+\boldsymbol{K}(\boldsymbol{\xi}, \boldsymbol{\xi})] \boldsymbol{i}=\boldsymbol{u}(t) \\
& \boldsymbol{F}(\boldsymbol{\xi})=m \ddot{\boldsymbol{q}} \\
& \boldsymbol{M}(\boldsymbol{\xi})=\boldsymbol{I}_{\theta \theta} \dot{\boldsymbol{\omega}}+\boldsymbol{\omega} \times \boldsymbol{I}_{\theta \theta} \boldsymbol{\omega}
\end{aligned}
$$

where $\boldsymbol{\xi}$ is a vector including the set of positions and orientations of the elementary volume at the instant $t$ and $\dot{\xi}$ is its derivative. It is worth to note that some of the currents included in $\boldsymbol{i}$ may be imposed. In order to improve the accuracy of the integration method, while retaining relatively long time steps, a prediction correction scheme is adopted.

The rationale behind it is the search for an approximation of the time dependence of the coefficients $\boldsymbol{L}(\boldsymbol{\xi})$ and $\boldsymbol{K}(\boldsymbol{\xi}, \dot{\boldsymbol{\xi}})$ in electrical equations and of the force and torque in the mechanical equations. The predictor-corrector approach is used to obtain an approximate behavior of the named quantities by a linear interpolation between the known values at the previous time step and the predicted values at the next time step. Inserting this knowledge in the equations has the effect of considering updated values of the coefficients, allowing a coupling between the equations, which is stronger than the one in a simply staggered scheme and comparable with a monolithic approach. The scheme can be summarized as follows $\left(\Delta t=t_{n+1}-t_{n}\right)$ :

a) assuming $\boldsymbol{L}(\boldsymbol{\xi})$ and $\boldsymbol{K}(\boldsymbol{\xi}, \dot{\boldsymbol{\xi}})$ as constant in $\Delta t$, currents are obtained by integrating (6a);

b) corresponding prediction of force and torque are evaluated and the forward Euler integration is used for (6b) and (6c) to obtain $\tilde{\boldsymbol{\xi}}_{n+1}$ an estimate of $\boldsymbol{\xi}$ at $t_{n+1}$;

c) a piecewise linear approximation is assumed for $\boldsymbol{L}$ and $\boldsymbol{K}$ in the interval $\left[t_{n}, t_{n+1}\right]$ :

$$
\begin{aligned}
& \boldsymbol{L}(t) \simeq \boldsymbol{L}\left(t_{n}\right)+\dot{\boldsymbol{L}}\left(t_{n}\right) \cdot\left(t-t_{n}\right)=\boldsymbol{L}_{n}+\dot{\boldsymbol{L}}_{n} \cdot\left(t-t_{n}\right), \text { with: } \\
& \dot{\boldsymbol{L}}\left(t_{n}\right)=\frac{\boldsymbol{L}\left(\tilde{\boldsymbol{\xi}}_{n+1}\right)-\boldsymbol{L}\left(\boldsymbol{\xi}_{n}\right)}{\Delta t} ;
\end{aligned}
$$

d) approximated expression for $\boldsymbol{L}$ and $\boldsymbol{K}$ are inserted in (6a) and the equation is integrated by a trapezoidal-like rule to obtain the corrected values of the currents at $t_{n+1}$;

e) finally, the corrected values of force and torque are evaluated and the corrected $\xi$ at $t_{n+1}$ is obtained.

An important reduction in computational time is achieved by using iterative methods for full matrices; furthermore, the solution at the $n$-th step is used as the initial estimate of the solution at the subsequent step. The analysis of massive 3D devices coupled with lumped external circuits is straightforwardly performed since the massive device is described by a network too.

\section{VALIDATION OF THE METHOD}

We validated the numerical formulation by comparison with the simple device shown in fig. 5. An analytical solution is available [14].

We performed two analysis; the first was characterized by: $L_{a, 1}=2.304 \mu \mathrm{H}, \quad L_{c, 1}=2.734 \mu \mathrm{H}, \quad M_{a c, 1, \max }=1.116 \mu \mathrm{H}$, $C R D_{1}=0.219(C R D$ is the differential compression ratio and $M_{a c, 1, \max }$ is the maximum value of the mutual induction coefficient between armature and compensating coils [14]).

The second had: $L_{a, 2}=2.304 \mu \mathrm{H}, \quad L_{c, 2}=2.432 \mu \mathrm{H}$ $M_{a c, 2, \max }=1.691 \mu \mathrm{H}$ with $C R D_{2}=0.686$. The angular speed of the rotating armature was $\omega=628 \mathrm{rad} / \mathrm{s}$; all the resistances were neglected and the load between the output terminals was zero (short circuit). Two versions of the analytical model were used. The difference was in the dependence of $M_{a c}$ with respect to $\vartheta$ (the angle between the axes of the coils). In the first $M_{a c}$ varies with the cosine of $\vartheta$ as in [14]. In the other we considered a more accurate dependence of $M_{a c}$ with $\vartheta$ as reported in fig 6 .

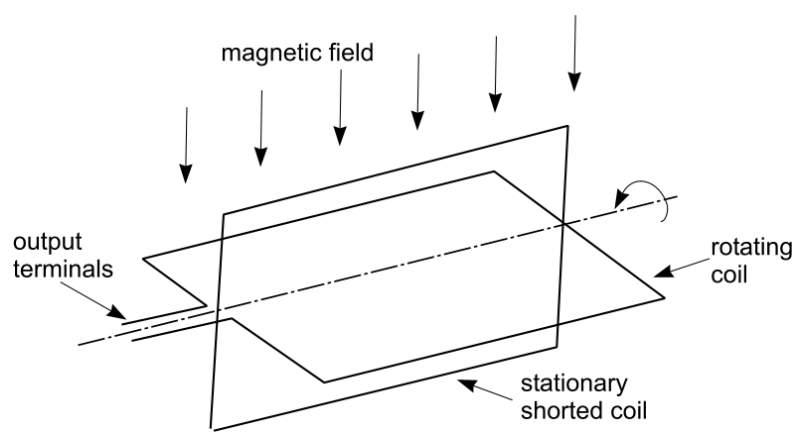

Fig. 5 The elementary compulsator as in [14].

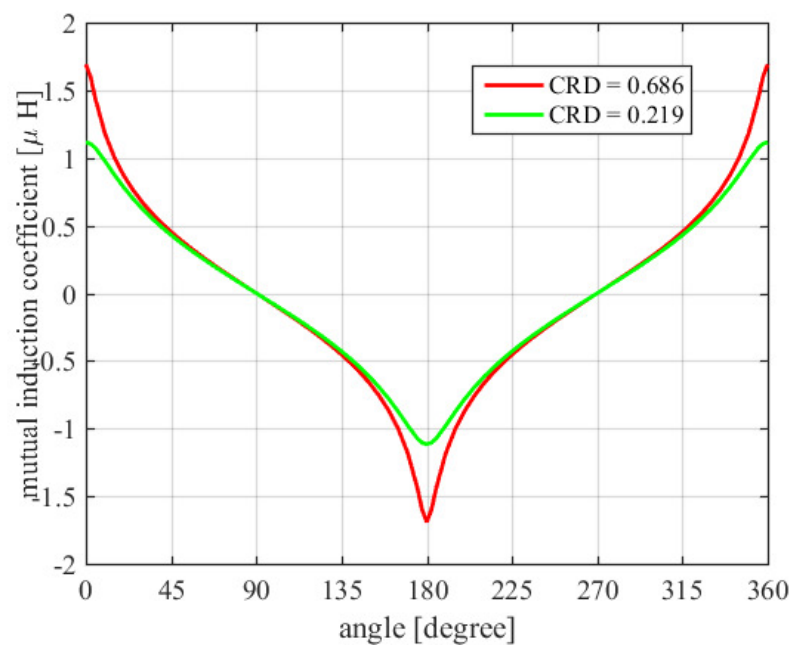

Fig. 6 Mutual inductance coefficients between armature and compensating coil as a function of the angle between the axes of the coils. 
The comparison between the results is reported in figs. 7 and 8. There is a substantial difference between the results obtained by the numerical analysis and the results reported in [14]. This is due to the strong deviation of the actual behaviour of $M_{a c}(\vartheta)$ (shown in fig. 6) with respect to the cosinusoidal waveform assumed in [14]. The difference is reduced if the analytical model is corrected by introducing the waveforms in fig.6. Considering that the numerical model performs a transient analysis, while the corrected analytical model reports the steady behaviour, the agreement between the respective results (red dashed and blue solid lines) is satisfactory.

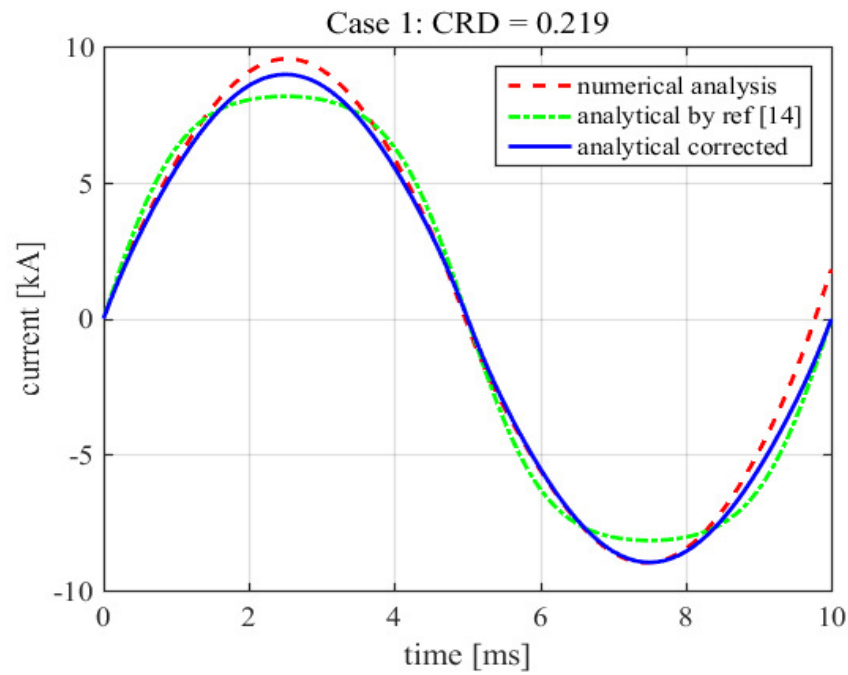

Fig. 7. Comparison between the numerical analysis and the analytical models.

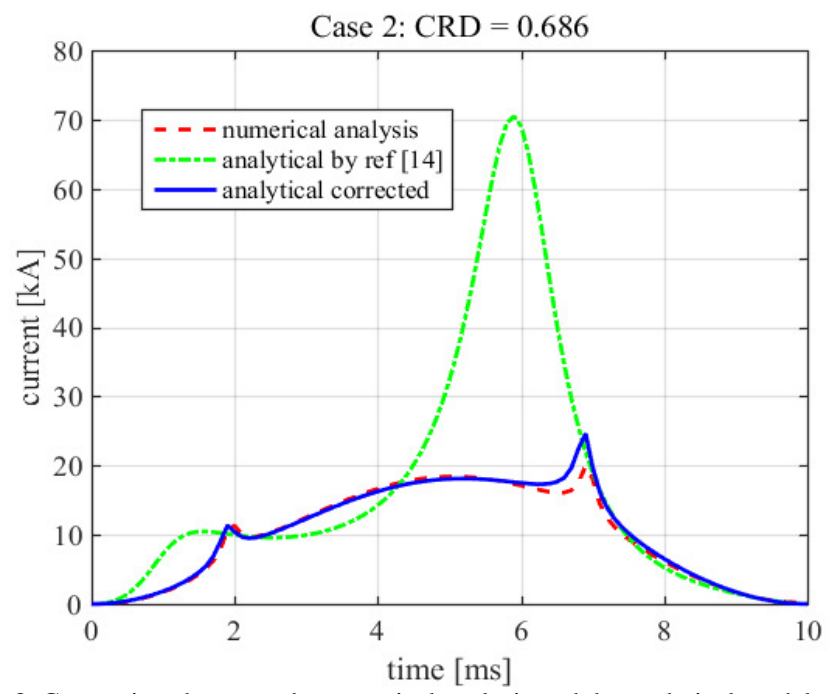

Fig. 8. Comparison between the numerical analysis and the analytical models.

\section{The SimUlated SySTEMS}

We analyzed a single-phase, two poles machine characterized by two different selective passive compensation. In the first, a discontinuous conductive shield is used as the compensating element, while in the second a set of single turns, each shorted on itself, is considered. The two machines share the same armature winding and the same filed coils; moreover, the active sides of the shorted turns occupy the same space as the discontinuous shield.

Both the analyzed devices are characterized by stationary field coils, which occupy the inner part of the stator, while the compensating components are located on the outer part of the stator. Armature windings are on the rotor, whose angular speed is $\omega=628 \mathrm{rad} / \mathrm{s}$. Fig. 9 shows a cross sectional view of the machine as modeled by EN4EM code. In the figure, the axis of the field coil is in the vertical direction, while the axis of the armature coil is horizontal. Fig. 10 shows a 3D view of one the two 25 turns field coils, capable of producing a magnetic flux density of about $2 \mathrm{~T}$ on the coils axis at the radius of the armature coils. The structure of the armature coils is the same as the field coils, but the number of turns, which is 6 for each armature coil.

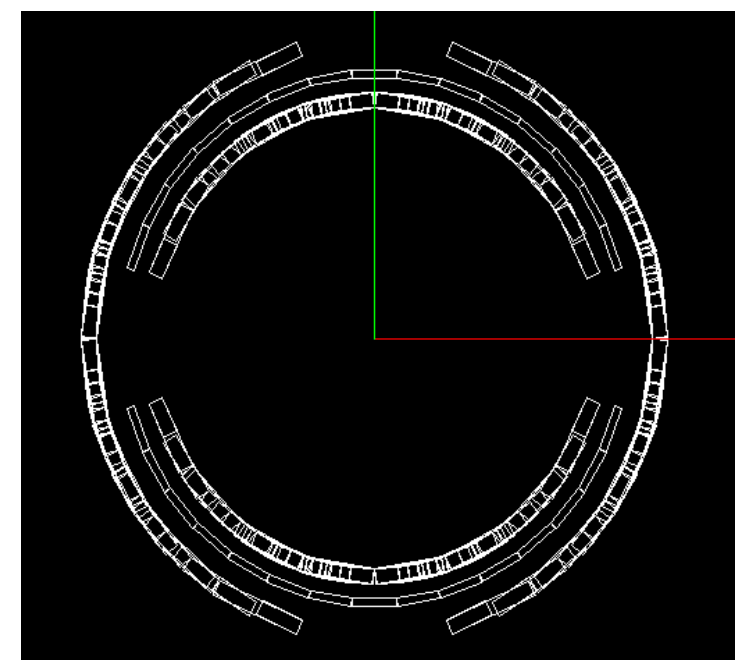

Fig. 9. Cross sectional view of the discretized device.

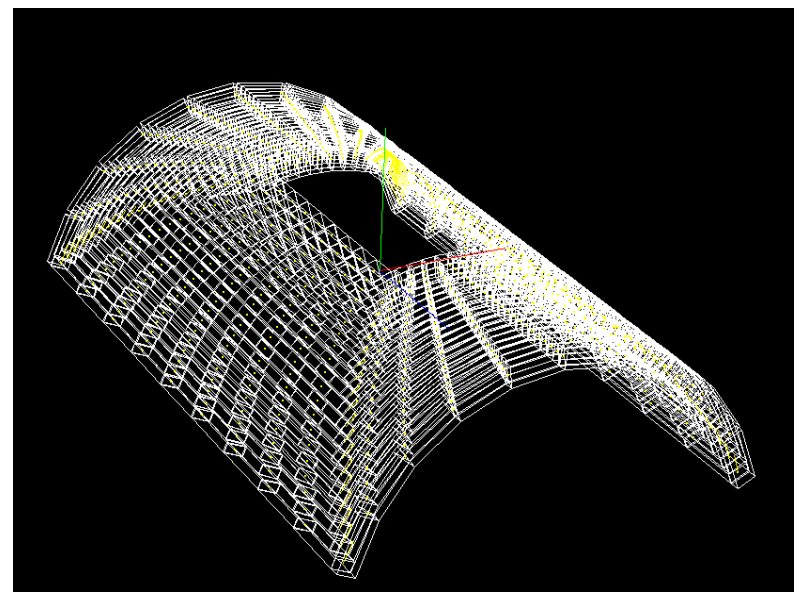

Fig. 10. Discretization of one of the two field coils. 


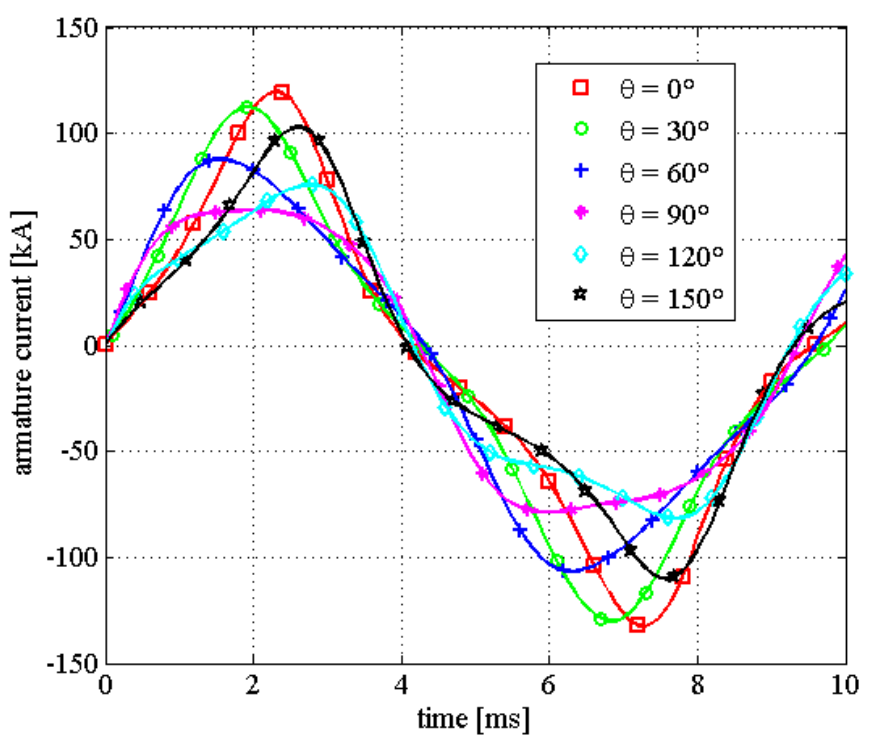

Fig. 11. Armature current for different angular position of the shield when armature and field coils are in quadrature at $\mathrm{t}=0$.

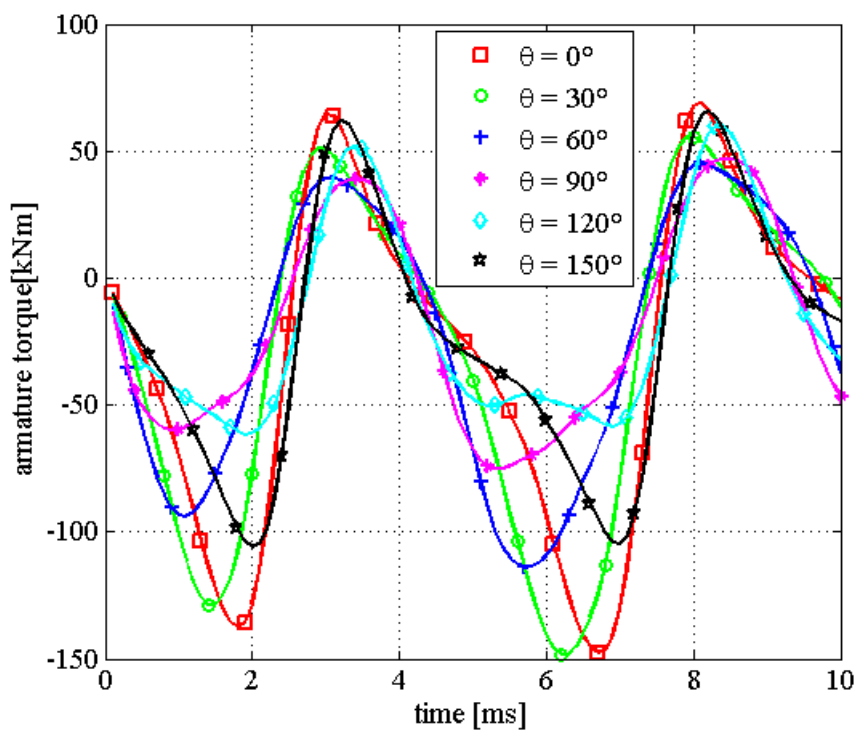

Fig. 12. Torque on the armature coils for different angular position of the shield when armature and field coils are in quadrature at $\mathrm{t}=0$.

The outer radius of the field coils is $16 \mathrm{~cm}$; the axial length is $0.5 \mathrm{~m}$ and they span an angle of 150 degrees.

In the first device the compensating shield consists of two aluminum sheets spanning an angle of 150 degrees each, with an inner radius of $17 \mathrm{~cm}$, a thickness of $5 \mathrm{~mm}$, an axial length of $0.5 \mathrm{~m}$. In the second, 15 diametrical shorted turns are located in the same position as the shield. They span the same angle and the radial thickness is $5 \mathrm{~mm}$. Cross sectional view is the same as the one shown in fig. 9.

The armature coils have an inner radius of $19 \mathrm{~cm}$, a thickness of

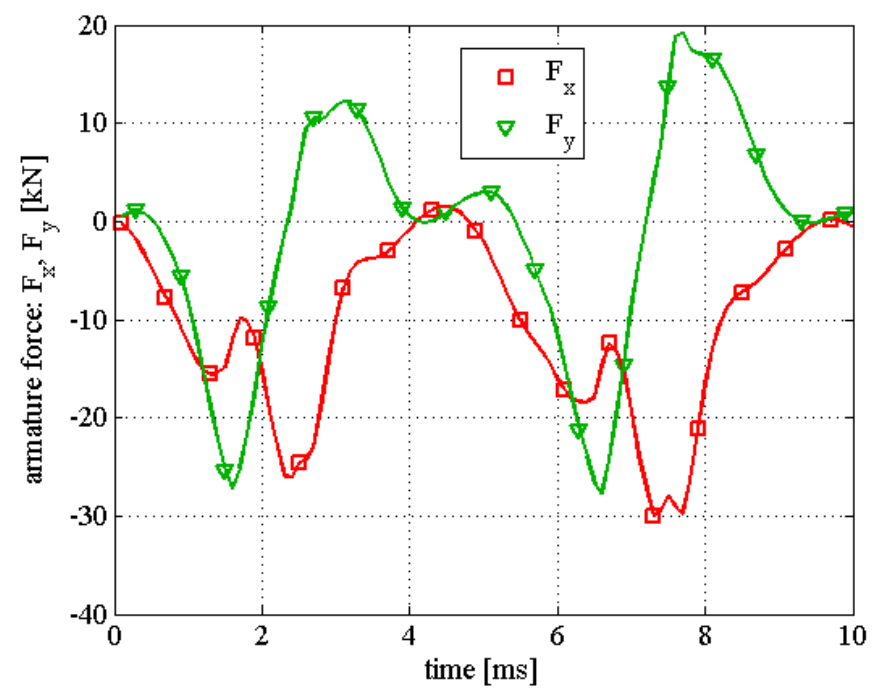

Fig. 13. Transverse force on the rotor when the shaft is $4 \mathrm{~mm}$ off-centered when $\vartheta=0$ and when armature and field coils are in quadrature at $\mathrm{t}=0$.

$1 \mathrm{~cm}$, and are constituted by 6 turns each; they are series connected and have been terminated on a load constituted by the series connection of $R_{l}=1.0 \mathrm{~m} \Omega$ and $L_{l}=1.0 \mu \mathrm{H}$.

We performed a number of simulations considering several relative angular positions of the shield with respect to the field coils for both the compensating solutions. We first considered the discontinuous compensating shield initiating the current pulse at the peak positive voltage induced on the armature (i.e. the axis of the field and armature coils are in quadrature as in fig. 9). Figure 11 shows the waveforms of the current on the load in correspondence of 6 angular positions spanning 150 degrees. In the first position, the shield discontinuity is aligned with the quadrature axis of the field coils as in fig. 9. This position is indicated by $\vartheta=0$. Subsequent positions are equally spaced and they increase in the opposite directions w.r.t. the motion of the armature, which rotates counterclockwise. We simulated the device for a time interval of $10 \mathrm{~ms}$, which is the period corresponding to $\omega=628 \mathrm{rad} / \mathrm{s}$, using a time step $\Delta t=0.1 \mathrm{~ms}$.

Fig. 12 shows the corresponding electromagnetic torques acting on rotor of the machine. As expected the torques have an impulsive behavior correspondent to the time interval where the current is delivered to the load.

With reference to the arrangement shown in fig. 9, we also performed a simulation with the rotor shaft off-centered of $4 \mathrm{~mm}$ in the horizontal direction. Fig. 13 shows the forces along the $\mathrm{x}$ and $y$ axes acting on the rotor. Because of these forces, vibrations on the structure are expected. Since the EN4EM code is able to integrate the 6 DoFs mechanical equation of the rigid body, the knowledge of the stiffness of the bearings allows to obtain the transverse displacement of the shaft. 


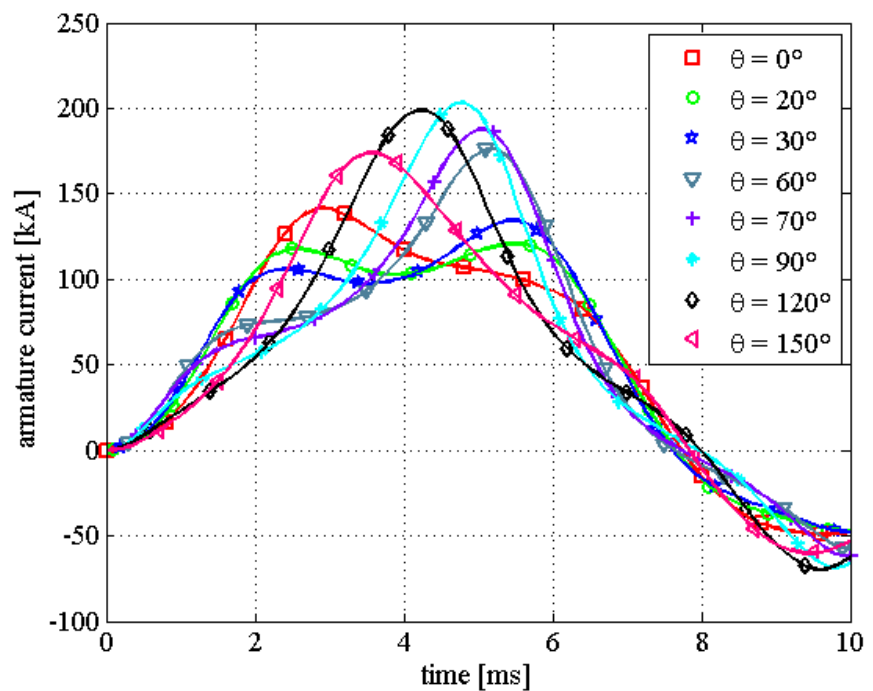

Fig. 14. Armature current for different angular position of the shield when armature and field coils are in phase at $\mathrm{t}=0$.

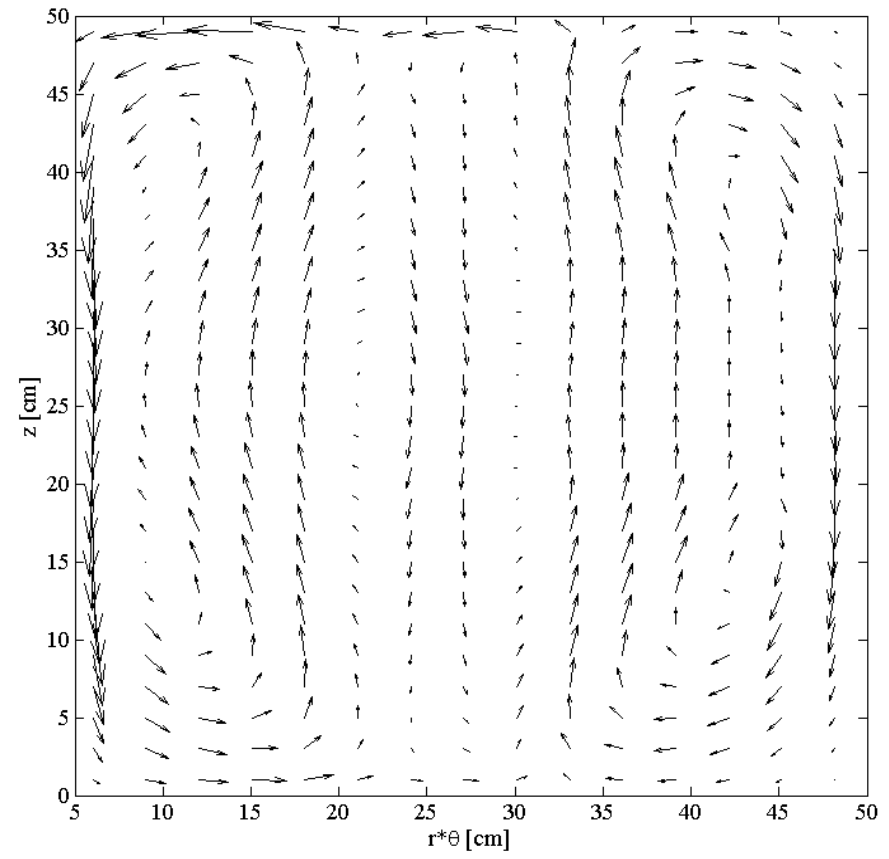

Fig. 15. Eddy current density distribution on the shield at $\mathrm{t}=50 \mathrm{~ms}$ when $\vartheta=0$ and when armature and field coils are in quadrature at the firing instant.

Another set of simulations was performed for the machine with the discontinuous compensating shield, initiating the current pulse at the instant of zero induced voltage on the armature. This happens when the field coils are rotated of $90^{\circ}$ with respect to the position shown in fig. 9. Fig. 14 shows the armature currents for different angular positions of the shield, while fig. 15 shows the current density distribution on the shield, at $t=5.0 \mathrm{~ms}$, and when $\vartheta=0$.

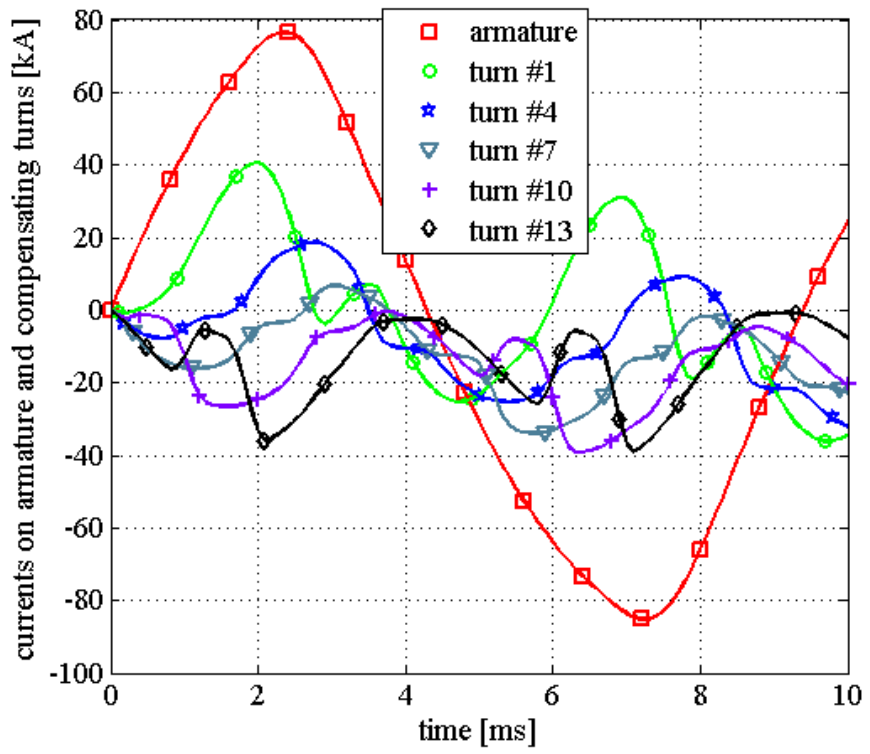

Fig. 16. Currents on the armature and on some compensating turns when armature and field coils are in quadrature at $\mathrm{t}=0$, and when compensating turns are as in fig. 9 .

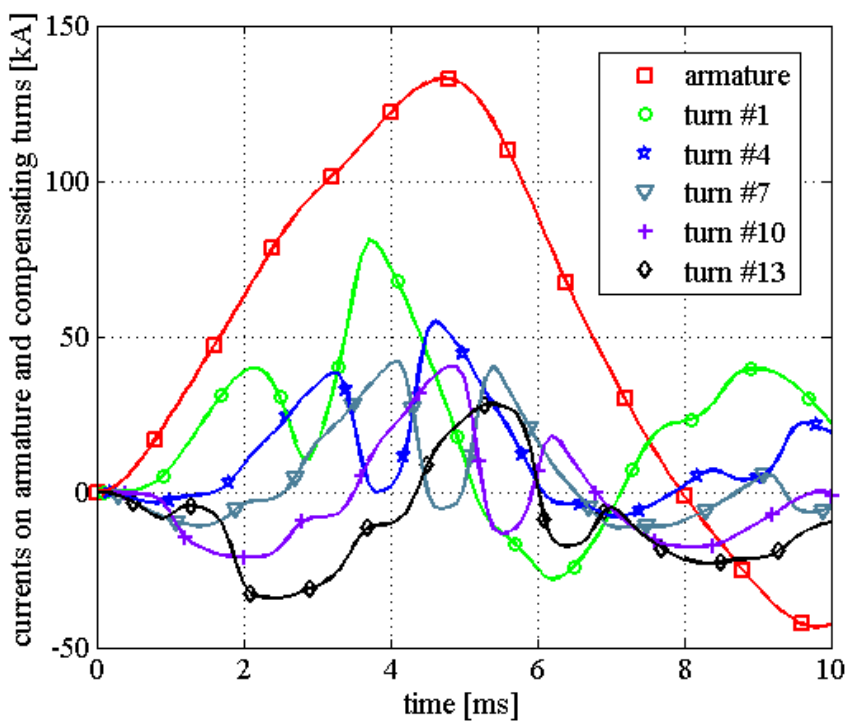

Fig. 17. Currents on the armature and on some compensating turns when armature and field coils are in phase at $\mathrm{t}=0$, and when compensating turns are as in fig. 9.

Since the penetration depth at the frequencies involved in the device operation $(100 \mathrm{~Hz})$ is greater than the thickness of the shield, we can assume the current density distribution as uniform in the radial direction.

Fig. 16 shows the current on the armature and the currents on some of the shorted compensating turns when the current pulse initiates in correspondence to the peak positive voltage, 


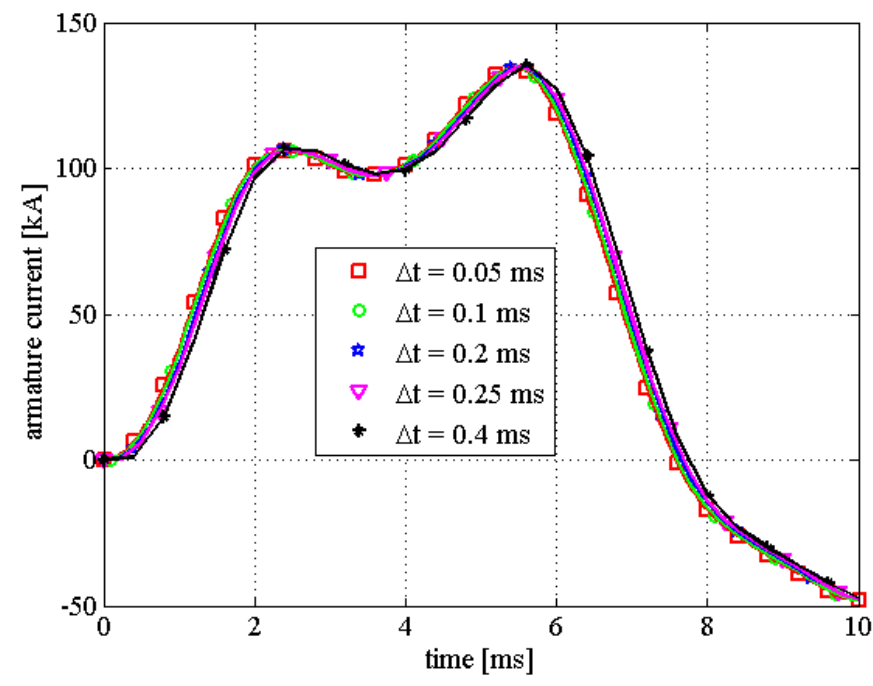

Fig. 18. Comparison of the results obtained by different time steps.

and the compensating turns are as in fig. 9. The compensating turns are numbered counterclockwise.

Fig. 17 reports the same quantities as in fig. 16 when the current pulse is initiated at zero voltage on the armature. The initial configuration is the one in fig. 9 with the fields coils rotated of $90^{\circ}$.

Finally, to test the robustness of the time integration scheme implemented in EN4EM code we performed several simulations of the same configuration with different time steps ranging from $\Delta t=0.05 \mathrm{~ms}$ to $\Delta t=0.4 \mathrm{~ms}$. The results, reported in fig. 18, show that the most evident effect is a small delay of the waveforms obtained with the longer time steps. Considering that the CPU time is proportional to the number of steps, the saving in computation time can be very relevant.

\section{CONCLUSION}

The research code EN4EM has proven to be effective in the numerical analysis of the air core compensated pulsed alternator. Two machines, one characterized by a selective passive compensation obtained by a discontinuous conductive shield and the other with a set of single shorted turns, each shorted on itself, have been investigated. The general behavior of the results agrees with experiments and theoretical studies described in literature [14], [15]. Finally, the robustness of the time integration scheme demonstrates the excellent performance of the code.

\section{ACKNOWLEDGMENT}

The authors would like to thank the NVIDIA's Academic Research Team for their equipment donation of two NVIDIA Tesla K20c GPUs that have been extensively used for the simulation activity.

\section{REFERENCES}

[1] J. R. Kitzmiller, S. B. Pratap, M. D. Driga, "An application guide for compulsators," IEEE Trans on Mag., vol.39, no.1, pp.285-288, Jan. 2003.

[2] M. L. Spann, S. B. Pratap, M. D. Werst, W. A. Walls, and W. G. Fulcher, "Compulsator research at The University of Texas at Austin-an overview," IEEE Trans. Magn., vol. 25, pp. 529-537, Jan. 1989.

[3] D. W. Wehrlen, J. H. Gully, "Small caliber mobile EML," IEEE Trans. Magn., vol. 22, no. 6, pp. 1804 - 1807, Nov. 1986

[4] M. L. Spann, S. B. Pratap, W. F. Weldon, W. A. Walls, "Rotating machines-power supply for the next generation of EM accelerator, IEEE Trans. Magn., vol. 27, no. 1, pp. 344 - 349, Jan. 1991.

[5] W. A. Walls and M. D. Driga, "Topologies for compact compensated pulsed alternators," Pulsed Power Plasma Sci., vol. 1, pp. 191-194, 2001.

[6] Y. Caiyong, Y. Kexun, L. Zhenxiu, and Y. Pan, "Investigation of pulseexcitation in pulse alternator system,"IEEE Trans. Plasma Sci., vol. 39, no. 1, pp. 342-345, Jan. 2011.

[7] Caiyong Ye; Kexun Yu; Hua Zhang; Pei Yuan; Qingming Xin; Jianbo Sun, "Comparison Between Self-Excitation and Pulse-Excitation in Air-Core Pulsed Alternator Systems," IEEE Trans. Plasma Sci, vol.41, no.5, pp.1243-1246, May 2013.

[8] Z. Weiduo, S. Wu, C. Shumei, W. Xuejiao, "Electromagnetic shields of the air-core compulsator," IEEE Trans. Plasma Sci., vol. 43 no. 5, pp. $1497-$ 1502, May 2015.

[9] S. Wu, S. Cui, L. Song, W. Zhao, and J. Zhang, "Design, simulation, and testing of a dual stator-winding all-air-core compulsator," IEEE Trans. Plasma Sci., vol. 39, no. 1, pp. 328-334, Jan. 2011.

[10] Q. Zhang, S. Wu, C. Yu, S. Cui, and L. Song, "Design of a model-scale air-core compulsator," IEEE Trans. Plasma Sci. vol. 39, no. 1, pp. 346353, Jan. 2011

[11] M. Crawford, J. A. Mallick, J. Pappas, "Use of SABER circuit simulation software for the modeling of compensated pulsed alternators driving a railgun load," IEEE Trans on Mag, vol.39, no.1, pp.337-342, Jan. 2003.

[12] A. Musolino, R. Rizzo, E. Tripodi and L. Toni, "Modeling of electromechanical devices by GPU-accelerated integral formulation." Int $J$. of Num. Mod.: Electronic Networks, Devices and Fields vol. 26, no. 4 pp. 376-396, 2013

[13] E Tripodi, A Musolino, R Rizzo, M Raugi, "A new predictor-corrector approach for the numerical integration of coupled electromechanical equations," Int. J. for Num. Meth..in Eng, in Press, DOI: 10.1002/nme.4974.

[14] S. B. Pratap, K. T. Hsieh, M. D. Driga, W. F. Weldon, "Advanced compulsators for railguns," IEEE Trans on Mag., vol. 25, n.1, pp.:454 459, Jan. 1989.

[15] M. D. Driga, S. B. Pratap, W. F. Weldon, "Advanced compulsator design," IEEE Trans on Mag., vol. 25, n.1, pp.:142 - 146, Jan. 1989. 